\title{
Investigation of the vortex ring transition using scanning Tomo- PIV
}

\author{
Zhengzhong $\operatorname{Sun}^{1}\left[\right.$ Christoph Brücker ${ }^{1}$
}

Received: 21 October 2016 / Revised: 22 February 2017 / Accepted: 23 February 2017 / Published online: 27 March 2017

(C) The Author(s) 2017. This article is an open access publication

\begin{abstract}
The transition of a vortex ring at $R e_{\Gamma}=5030$ is studied by time-resolved scanning tomographic PIV technique. The transition process is first analyzed through flow quantities such as circulation and vorticity components. Using the volumetric measurement technique, vortical organization of the vortex ring at early and late transition stages is visualized, respectively. Focus is paid to the instability phenomenon associated with transition. The present $4 \mathrm{D}$ flow data allows analysis of the temporal evolution of the wavenumber spectra. The dominant wavenumbers in transition are identified and the growth of their amplitude is revealed. The vortex ring transition is finally studied through the particle trajectories. A phase difference between the axial velocity and radial velocity is found at the beginning of transition; however, it is subject to change following the progression of transition. Statistical analysis on the velocity components helps to identify the aft portion of the inner ring as the one that is first to lose the original phase relation in velocity, which is caused by the secondary vortical activity during transition.
\end{abstract}

\section{Introduction}

Vortex ring is of fascinating nature in fluid dynamics. Both its formation and evolution have attracted numerous experimental (Maxworthy 1972; Widnall and Sullivan 1973; Gharib et al. 1998) and numerical studies (Bergdore et al. 2007; Archer et al. 2008; Ran and Colonius 2009). The

Zhengzhong Sun

zhengzhong.sun@city.ac.uk

1 Department of Mechanical Engineering and Aeronautics, City, University of London, London EC1V 0HB, UK vortex ring formation through a piston-cylinder mechanism has been investigated in detail by Gharib et al. (1998). The flow field of a vortex ring was found to be determined by the formation number (stroke length to piston diameter ratio). A threshold formation number was suggested. A trailing jet is attached to the leading vortex ring when produced at a formation number larger than the threshold. However, the vortex ring detaches from the trailing jet under a formation number smaller than the threshold value. Apart from the topic on formation, the evolution of vortex ring offers an opportunity to study the fundamental aspect of laminar-turbulent transition process. More recently, due to the increasing attention paid on the jet noise produced by airliners, the vortex ring is chosen as a conceptual model to analyze the noise generation (Ran and Colonius 2009), because it isolates the ring transition from the pairing procedure and provides insight on the noise associated solely with laminar-turbulent transition. In the present paper, focus is going to be paid on the transition behavior of a single vortex ring.

One early experimental study on the evolution of vortex ring was reported by Krutzsch (2011) in the 1930s. In that experiment, observations on the vortex ring transition were made with the then novel dye visualization technique, which is in use even nowadays in vortex ring studies (New et al. 2016). The laminar circular ring was observed to exhibit wavy undulations along the core through instability mechanism. Moreover, secondary structures associated with this flow instability were identified, too. Although this early work only provided qualitative observation, it did pave the foundation for the topic of vortex ring transition and guided research efforts towards the instability phenomenon in the vortex ring transition.

Development of the PIV technique in the last three decades makes quantitative examination on the vortex ring 
transition possible, allowing advanced instability analysis based on the characteristic flow field. The experimental study of Dazin et al. (2006) using the stereoscopic PIV technique successfully revealed the transition of vortex rings produced at various conditions with piston-based Reynolds number in the range of $R e_{\mathrm{p}}=2600 \sim 5100$. Flow structure that appears during transition was clearly captured. For example, pockets of high concentration of radial velocity are produced along the ring torus, while the number of pockets is linked with the wave number. Spectrum analysis on the unsteady radial velocity was also performed at a few snapshots, providing the most unstable mode for the instability. The phase angle of the unstable modes in transition was also revealed to remain unchanged, which confirms the standing-wave nature of the instability growing at ring torus. Despite the success of applying the stereo-PIV technique into the analysis of instability phenomenon, the temporal evolution of the flow instability was not able to be captured as the laser sheet was fixed to a certain location. A recent experimental work by Pointz et al. (2016) enables tracking of the vortex ring motion by translating the flow facility in the direction counter to the ring travelling direction. Together with the scanning tomographic PIV technique, this unique facility allowed to take $4 \mathrm{D}$ measurement of the vortex ring transition process. Taking advantage of the 4D data ensemble, dynamic modal analysis was used to reveal the primary vortical structure produced in transition. Apart from the wavy ring torus, the halo-vortex was identified to wrap around the ring core. However, attention was not paid in the flow instability associated with transition. Therefore, the first motivation of the present experimental work is to reveal the temporal growth of the instability phenomena.

The development of computational fluid dynamics methodology enables the direct numerical simulation (DNS) of the vortex ring transition. The work of Archer et al. (2008) simulated the evolution of vortex ring under a few circulation-based Reynolds numbers, namely $R e_{\Gamma}=3000 \sim 10,000$. As the DNS result offers volumetric information, the revealed complete vortical structure at transition agrees with that visualized through the scanning Tomo-PIV technique (Pointz et al. 2016). Taking advantage of the timeresolved feature, the amplitude growth of the unsteady modes was studied, which eventually facilitates comparison with prediction form theory. Analysis of the fluid trajectories was finally provided to reveal the fluid entrainment and detrainment during transition. The transition in a Lagrangian point of view has unfortunately not been provided by experimentalists. The second motivation is thus to analyze the transition through a Lagrangian point of view.

The present experimental work will take full advantage of the facility presented by Pointz et al. (2016). The use of the scanning Tomo-PIV will result in a time-resolved three-dimensional data set, which is able to address the motivations described above. The experimental setup is briefly presented in the next section. The transition procedure of the vortex ring is first summarized through a few quantities, such as circulation and vorticity components. Upon knowing the characteristics of the transition process, the growth of the instability activity is analyzed. The transition is afterwards studied through the Lagrangian point of view by means of 3D particle tracking. The conclusions are finally made at the end of the paper.

\section{Experimental setup}

The experimental setup as shown in Fig. 1 follows that in the experiment of Pointz et al. (2016) and it is only briefly discussed here. The measurement is carried out in a water tank with octagonal cross section. The present vortex ring is generated through a piston-cylinder nozzle installed at the top of the tank. The nozzle outlet has a diameter of $30 \mathrm{~mm}$. According to the formation number for vortex ring generation provided by Gharib et al. (1998), a small formation number less than 4 is selected to avoid the railing jet. The vortex ring travels down towards the bottom of the tank. The water tank is lifted upward at a constant speed to keep the vortex ring within the field of views of the static imaging system. A separate visualization experiment is performed and finds that the travelling speed of the vortex ring is about $50 \mathrm{~mm} / \mathrm{s}$, which is set as the traversing speed of the tank. The benefit of the lifting-tank facility is twofold. It not only allows

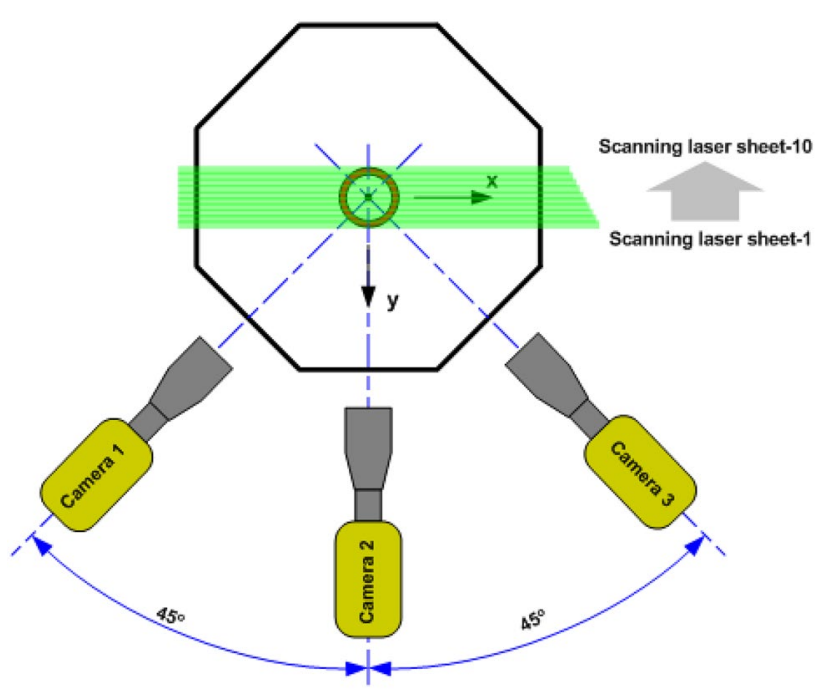

Fig. 1 Schematic of the experimental setup (topview) including the water tank, three-camera imaging system, and the scanning laser sheets 
the tracking of the vortex ring, but also avoids the vibration of the tomography imaging system.

The vortex ring is measured through the scanning Tomo-PIV, which has demonstrated fidelity in revealing the three-dimensional low-speed fluid flow (Pointz et al. 2016; Hegner et al. 2015). The imaging system is comprised of three Phantom V12.1 high-speed cameras with CMOS sensor of $1200 \times 800$ pixels. Each camera is equipped with telecentric lens (Sill Optics). The use of telecentric lens offers parallel line of sight and is advantageous for the back-projection procedure in volumetric intensity field reconstruction. The lens aperture is set $f_{\#}=1 / 16$, allowing a depth of focus of about $10 \mathrm{~cm}$. The imaging magnification factor is later determined to be 0.2 . The three cameras are arranged in a linear configuration with angular displacement of $45^{\circ}$ between each two neighboring cameras. They are synchronized and operate at a frame rate of $1250 \mathrm{fps}$. A continuous wave Argon-Ion laser (Coherent Innova 70C, $3 \mathrm{~W}$ power at $528 \mathrm{~nm}$ wavelength) is used as illumination source. The output laser beam is about $1.5 \mathrm{~mm}$ in diameter and is further expanded to a diameter of $8 \mathrm{~mm}$. The present volume illumination is different from that used in the standard Tomo-PIV. Instead, it is achieved through a rotating drum scanner, which was originally developed by Brücker (1997). Ten mirrors are installed on the rotating drum with the same circumferential distance, while they are staggered equally along the drum axis. The resulting illumination is thus formed by ten parallel marching slices. The thickness of each slice is determined by the laser beam, hence $8 \mathrm{~mm}$. An overlap of $2 \mathrm{~mm}$ is intentionally arranged for the neighboring slices, and the final volume has a thickness of about $62 \mathrm{~mm}$. The camera is synchronized with the scanner, each slice scan takes a period of $1 / 1250 \mathrm{~s}$, and the entire volumetric scan takes $1 / 125 \mathrm{~s}$, resulting in a volume imaging rate of $125 \mathrm{~Hz}$ for the 3D vortex ring, which is sufficient in resolving its evolution. Neutrally buoyant particles with a nominal diameter of $60 \mu \mathrm{m}$ are chosen as flow tracer. The entire internal memory of the camera ( $8 \mathrm{~GB}$ ), allowing acquisition of 8000 snapshots, is used for image recording. The total length of image acquisition then lasts $6.4 \mathrm{~s}$ at $1250 \mathrm{~Hz}$. Since the life time of vortex ring from formation to final turbulent breakdown lasts longer than the measurement duration, the imaging trigger is sent shortly after the ring is fully formed, such that the transition process can be captured.

The image data post-processing contains intensity volume reconstruction and vector calculation. Reconstruction is done through back-projection procedure as reported by Pointz et al. (2012) in detail. The reconstructed 3D intensity volume has size of $700 \times 519 \times 500$ voxels, with spatial resolution of $0.1 \mathrm{~mm} /$ voxel. 3D crosscorrelation of successive volumes is used to calculate the vectors, and the present $3 \mathrm{D}$ cross-correlation follows that of VODIM (Volume Deformation Iterative Multigrid) method (Scarano and Poelma 2009). The final interrogation volume has a size of $40 \times 40 \times 40$ voxels and processing is done on a grid with $75 \%$ overlap ratio. The resulting vector grid then has a spacing of $1 \mathrm{~mm}$ in all directions. The uncertainty of tomographic PIV is primary associated with the cross-correlation procedure, which is commonly estimated to be 0.1 voxel, corresponding to about $1.25 \mathrm{~mm} / \mathrm{s}$. The major parameters of the Tomo-PIV experiment are summarized in Table 1.

\section{Results and discussions}

\subsection{Characteristics of the vortex ring}

The characteristics of the present vortex ring is first discussed so that the initial condition for the follow-up evolution is known. The geometry of a vortex ring is usually described by the ring radius $R$ and the core radius $\delta$. The ring radius is defined as the location of maximum azimuthal vorticity along ring radius, as shown in Fig. 2 . The present ring radius is $R=22.5 \mathrm{~mm}$. The core radius is defined as the distance between the core centre and the location of maximum axial velocity. The core radius for the present ring is $\delta=8.5 \mathrm{~mm}$ and its slenderness ratio can be determined $\varepsilon=\delta / R=0.37$. The distributions of the azimuthal vorticity for a thicker $(\varepsilon=0.41)$ and thinner $(\varepsilon=0.20)$ ring, respectively, are also compared in Fig. 2 . These two rings are from the DNS study of Archer et al. (2008) and they both evolve from an initial Gaussian vorticity distribution. The azimuthal vorticity within the ring torus $(r / R<1.0)$ is reasonably bounded by the profiles of the other two rings due to its intermediate slenderness ratio. However, the trend is lost beyond the point of $r / R=1.25$. This could be caused by the viscous diffusion as the freshly formed ring is not captured in the present measurement.

Table 1 Parameters of Tomo-PIV experiment

\begin{tabular}{ll}
\hline Parameter & Quantity \\
\hline Numerical aperture $\left(f_{\#}\right)$ & $1 / 16$ \\
Volume sampling rate & $125 \mathrm{~Hz}$ \\
Magnification $(M)$ & 0.2 \\
Measurement volume & $70 \times 52 \times 50 \mathrm{~mm}^{3}$ \\
Voxel volume size & $700 \times 519 \times 500$ \\
Final interrogation volume & $40 \times 40 \times 40$ \\
& voxels $(75 \%$ \\
Number of vectors & overlap) \\
Vector spacing & $66 \times 48 \times 46$ \\
\hline
\end{tabular}




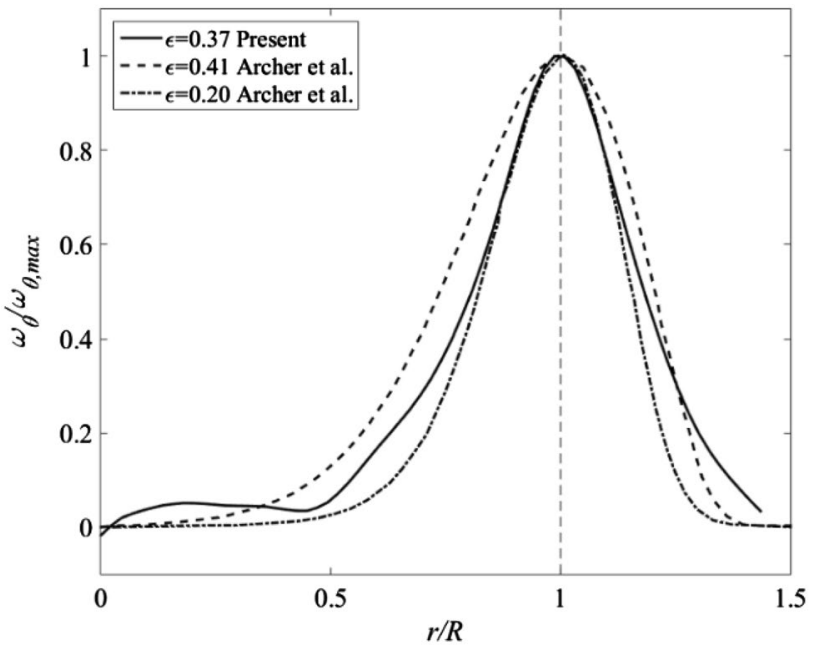

Fig. 2 Radial distribution of azimuthal vorticity component $\omega_{\theta}$

The initial vortex ring is represented through the two cross-sectional planes colored by azimuthal vorticity, as shown in Fig. 3. One plane goes through $z / R=0$, it is also named the median plane. A smooth ring structure is evidently visualized by the concentration of $\omega_{\theta}$. Note that the front and rear portions of the ring at both ends of $y$ axis are slightly cropped due to the limits of the scanning depth. The other plane is chosen at $y / R=0$, where regions of focused $\omega_{\theta}$ around the core region are revealed in symmetric arrangement relative to the ring axis. After a thorough inspection of initial vortex ring, its characteristic parameters are summarized in Table 2 . Note that the calculation of ring circulation follows $\Gamma=\int \omega_{\theta} \mathrm{d} r \mathrm{~d} z$. The circulation is studied in the next section to feature the ring transition process.

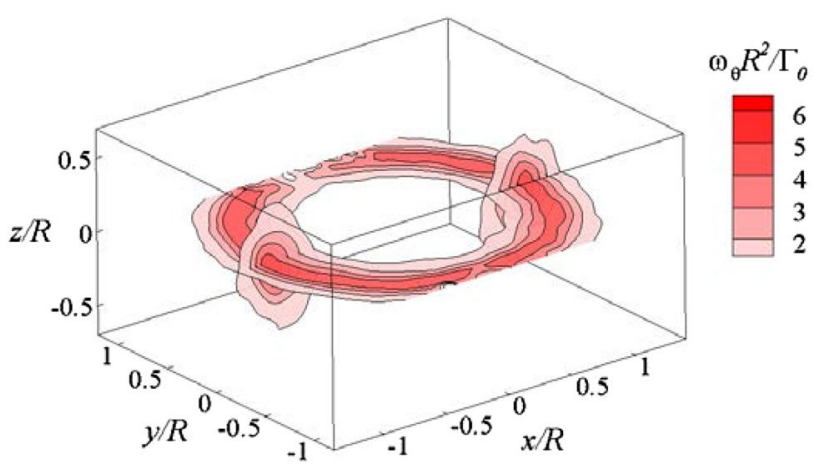

Fig. 3 Vortex ring in the first snapshot represented through two cross-sectional planes at $z=0$ and $y=0$. The two planes are colored by nondimensional azimuthal vorticity $\omega_{\theta} R^{2} / \Gamma_{0}$
Table 2 Characteristics parameters of the vortex ring

\begin{tabular}{ll}
\hline Parameter & Quantity \\
\hline Piston velocity & $155 \mathrm{~mm} / \mathrm{s}$ \\
Nozzle outlet diameter & $30 \mathrm{~mm}$ \\
Ring radius $(R)$ & $22.5 \mathrm{~mm}$ \\
Torus radius $(\delta)$ & $8.5 \mathrm{~mm}$ \\
Slenderness ratio $(\varepsilon)$ & 0.37 \\
Circulation $\left(\Gamma_{0}\right)$ & $5032.6 \mathrm{~mm}^{2} / \mathrm{s}$ \\
Piston-based Reynolds number $\left(R e_{\mathrm{p}}\right)$ & 4650 \\
Circulation-based Reynolds number $\left(R e_{\Gamma}\right)$ & 5030 \\
\hline
\end{tabular}

\subsection{Evolution of circulation and vorticity components}

The overall evolution of the present vortex ring can be represented through a few quantities. The start of the measurement is chosen to be $t^{*}=\frac{t \Gamma_{0}}{R^{2}}=0$. Note that the piston motion has started earlier and the recording is triggered to start after the roll-up process of the shear layer has finished. The first 12 snapshots of the present measurement, corresponding to $t^{*}<1$ belong to the steady ring whose circulation is conserved after the vortex ring formation, see Fig. 4a. This circulation-conservation behavior of the vortex ring was well revealed in the work of Gharib et al. (1998). Following the circulation plateau, the ring circulation is subject to a slow decay until about $t^{*}=50$ when $70 \%$ of original magnitude $\Gamma_{0}$ remains. Fitting the circulation decay into a power law $\left(\Gamma \sim t^{c}\right)$ finds that the power law exponent $c$ is -0.046 . The circulation decay of vortex ring with Reynolds number between 2000 and 4000 was studied by Dabiri and Gharib (2004), the curve fitting using power law returned exponent $c=-0.27 \sim-0.067$. The current less steep decay can be attributed to the slightly larger Reynolds number, because a higher Reynolds number hinders viscous diffusion. A smaller power law exponent (measured in absolute magnitude $|c|)$ was also reported in the DNS study of vortex ring at higher Reynolds number by Archer et al. (2008). The final phase of circulation evolution features a rapid drop in circulation intensity, with about $25 \%$ of $\Gamma_{0}$ left until the end of the measurement. This steep change is associated with turbulent activity, more efficient in dissipating circulation.

The axial velocity at the ring centroid is another useful parameter in revealing the evolution process. Similar as circulation, the initial phase when $t^{*}<1$ has near constant $w_{\underline{\underline{c}}}$, see Fig. 4c. The decay of centroid velocity is different from that of circulation, and it is subject to three stages. The first stage decay, namely, $t^{*}=1 \sim 35$, has a very subtle decrease, suggesting that the vortex ring travels at relatively steady speed in this phase. A much faster reduction in $w_{\mathrm{c}}$ takes place later until $t^{*}=58$, where the centroid axial velocity is only $\frac{w_{c} R}{\Gamma_{0}}=0.1$. The faster intensity decay is associated with 

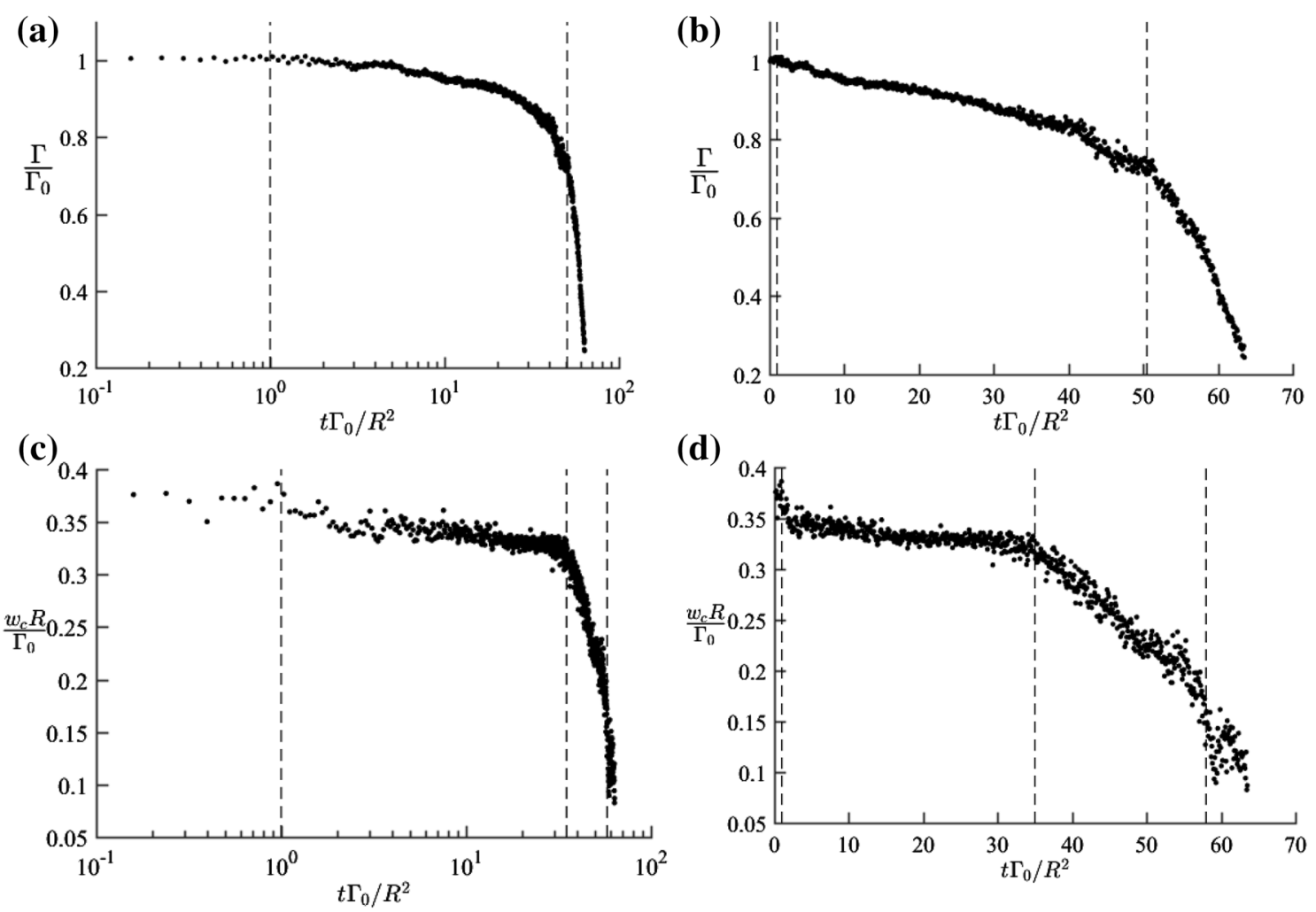

Fig. 4 Temporal evolution of circulation (a, b) and axial velocity at ring centroid (c, d). Left logarithmic $x$-axis scaling; right regular equidistant $x$-axis scaling

the secondary vortices generated in the transition. In the end, namely, the fully turbulent stage, the ring is not able to induce coherent motion, and the axial velocity stays low.

It has been extensively shown that there is generation of axial and radial vorticity in the transition process (Archer et al. 2008; Dazin et al. 2006). The three vorticity components in cylindrical coordinate systems for the present vortex ring are discussed. The evolution of vorticity components is presented through the volume weighted total vorticity magnitude $\bar{\omega}$ as shown in Fig. 5 . The evolution of azimuthal vorticity is similar as that of circulation, which is not surprising as the calculation of circulation is based on the azimuthal vorticity component. Growth of radial and axial components happens almost simultaneously at $t^{*}=20$, which indicates the beginning of transition. In the following temporal range of $t^{*}=20 \sim 50$, both $\omega_{r}$ and $\omega_{z}$ are subject to significant growth although at slightly different growth rates. The peak of radial vorticity is reached at $t^{*}=48$, however, that of axial component is achieved earlier at about $t^{*}=42$. Decay of both components happens as soon as reaching their peak values. Relating with the observations made in Fig. 4, the temporal range when axial and radial vorticity lose intensity also features decay of circulation and centroid axial velocity. So far, the transition of present vortex ring takes place within $t^{*}=20 \sim 50$, while

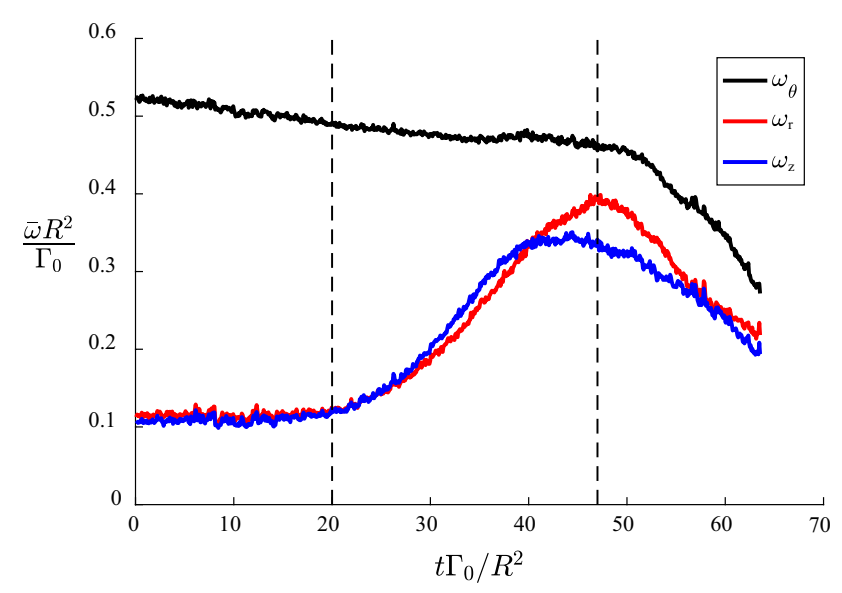

Fig. 5 Evolution of vorticity components in cylindrical coordinate

laminar $\left(t^{*}<20\right)$ and early turbulent $\left(t^{*}>50\right)$ phases are also captured in the present measurement.

\subsection{Vortical structure in transition}

The transition process of the present vortex ring is now visualized through vortex identification method using the Q-criterion. The vortex rings in six snapshots are shown 


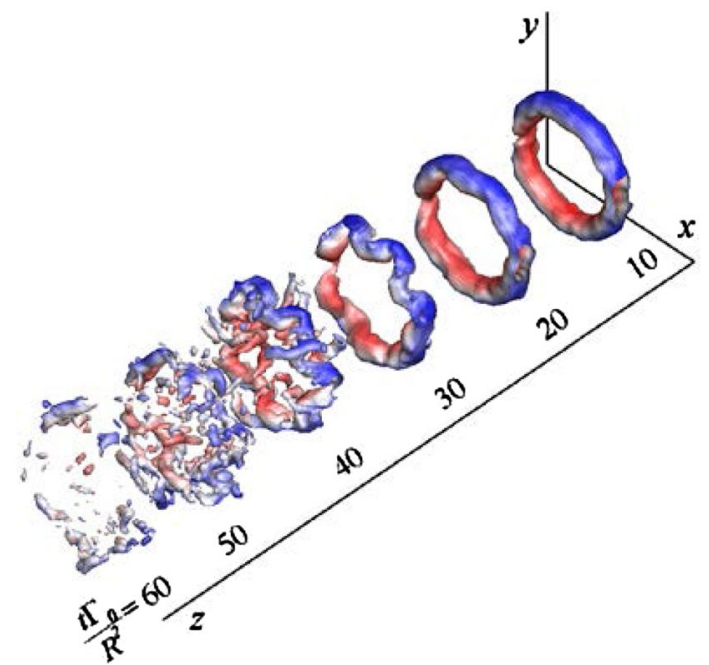

Fig. 6 Evolution of the vortex ring revealed by $Q=7$, and the isosurfaces are colored by axial velocity magnitude

in Fig. 6, and they are represented by isosurfaces of $Q=7$ from $t^{*}=10 \sim 60$. Note that the isosurfaces are colored by the axial velocity. The ring at $t^{*}=10$ belongs to the laminar phase and it shows up as a smooth torus. At the early transition stage from $t^{*}=20 \sim 30$, development of wave begins to take place, which is the characteristic phenomenon of the early phase of transition. The growth of the wave amplitude is rather clear in the present visualization. It terminates when the torus tilts $45^{\circ}$ from its original orientation, which has been visualized by various authors (Archer et al. 2008; Pointz et al. 2016). In the linear phase, the generation of axial and radial vorticity components is caused by the undulation of the ring torus. The nonlinear transition phase follows immediately and it is featured by the generation of secondary vortical structures in addition to the ring torus. Substantial secondary vortical structures can be observed in the ring at $t^{*}=40$. These vortex filaments develop in axial and radial directions and they are in the region surrounding the undulated torus. Recalling the growth of axial and radial vorticity magnitude in Fig. 5, the steeper growth rate of the two components after $t^{*}=30$ can be explained by the generation of the secondary vortices. As soon as the transition finishes, the ring enters turbulent phase, in which the coherent vortical structure breaks up into smaller eddies, and is subject to rapid dissipation. The vortices at $t^{*}=50$, namely, at early turbulent stage, is subject to very fast decay and already becomes weak eddies at $t^{*}=60$.

The two transition stages are going to be explored separately. The ring at $t^{*}=20$ is visualized through isosurface of $Q=14$ in Fig. 7. The number of waves developed on a ring depends on the aspect ratio. According to the empirical estimation provided by Shariff et al. (1994), the present ring with slenderness ratio of 0.37 is predicted to develop

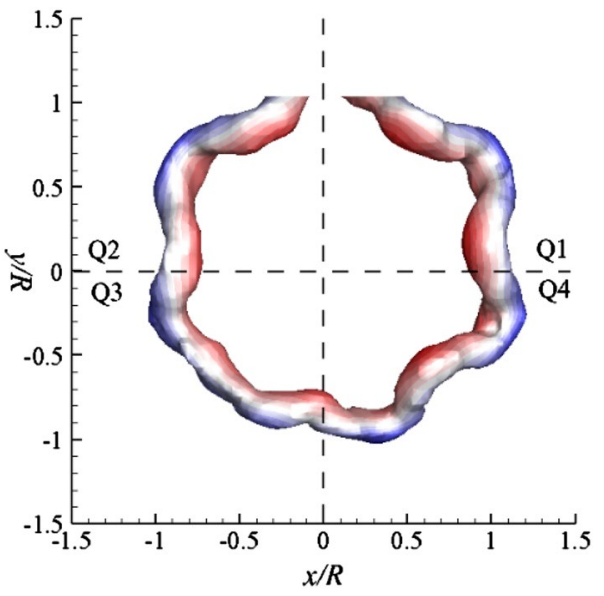

Fig. 7 Top view of vortex ring at $t^{*}=20$ visualized by $Q=14$

azimuthal instabilities with a primary wavenumber of 6 . The waves in the first, second, and fourth quadrants are of nearly the same wavelength, while that in the third quadrant has much smaller wavelength. This is the result of two competing wavenumbers in the azimuthal instability, and the bimodal feature has also been reported by Saffman (1978). The primary and secondary wave numbers will be analyzed in the next section. The vortex ring in the late transition stage at $t^{*}=40$ is shown in Fig. 8 through isosurface of $Q=3.5$, with which the undulated torus and secondary vortices are visualized simultaneously with the latter wrapping around the former. In the region within the ring, secondary vortices are aligned in axial direction, but they become tilted against the vortex axis in the region outside the torus. Again, due to the effect of two competing wavenumbers, the secondary vortical structural in the third quadrant in the top view (Fig. 8a) is not as well defined as in the other locations.

The evolution of the axial vorticity component within the median plane (the $x-y$ plane going through $z=0$ ) is representative of the transition process, because both the undulated ring torus and secondary vortices leave footprints there. The axial vorticity component in the median plane at three typical snapshots of $t^{*}=30,40$, and 50 are shown in Fig. 9. At the early transition stage $t^{*}=30$, axial vorticity patterns with alternating signs are produced along concentric layers. Due to the existence of competing wave numbers, this vorticity pattern is not well-defined in the bottom left region, which manifest in shortened wave numbers as observed in Fig. 7. The DNS work of Archer et al. (2008) revealed a similar effect in the contour of axial vorticity in the median plane, where vorticity pockets are produced with weakened intensity and smaller separation at one corner. The circular layer at $r / R=1.0$ belongs to the undulated ring core while the inner layer with weaker intensity is the cross-section of the axial secondary filaments. The 

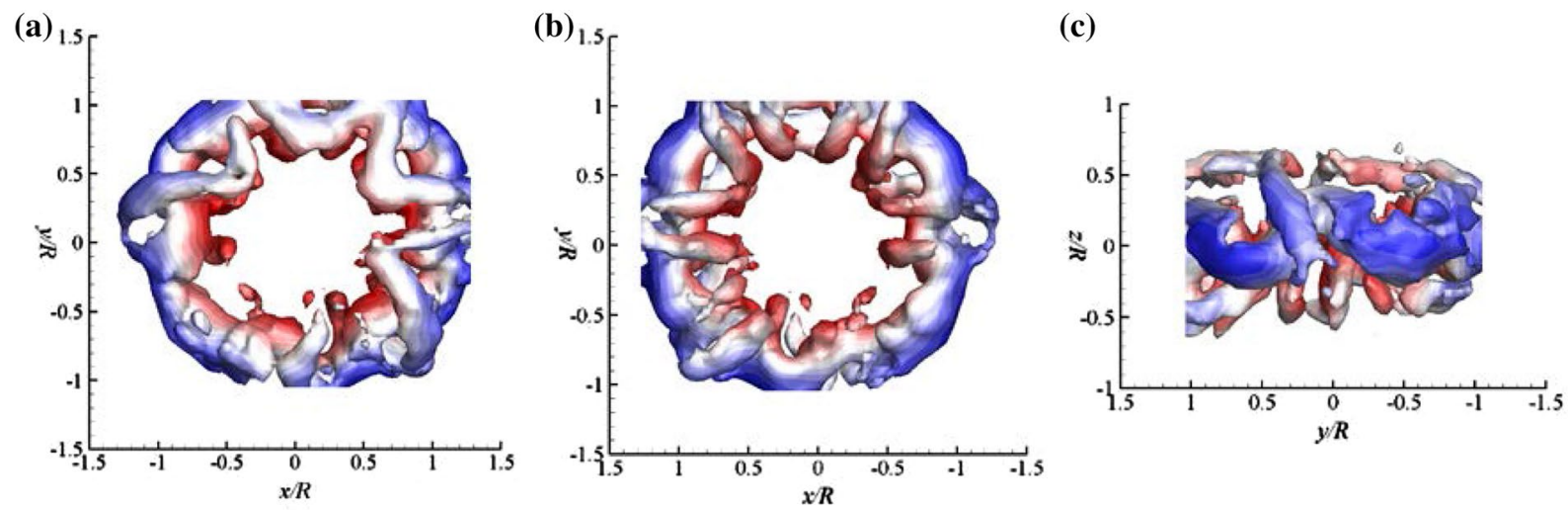

Fig. 8 Vortex ring at $t^{*}=40$ through isosurface of $Q=3.5$ : a top view, $\mathbf{b}$ bottom view, $\mathbf{c}$ side view
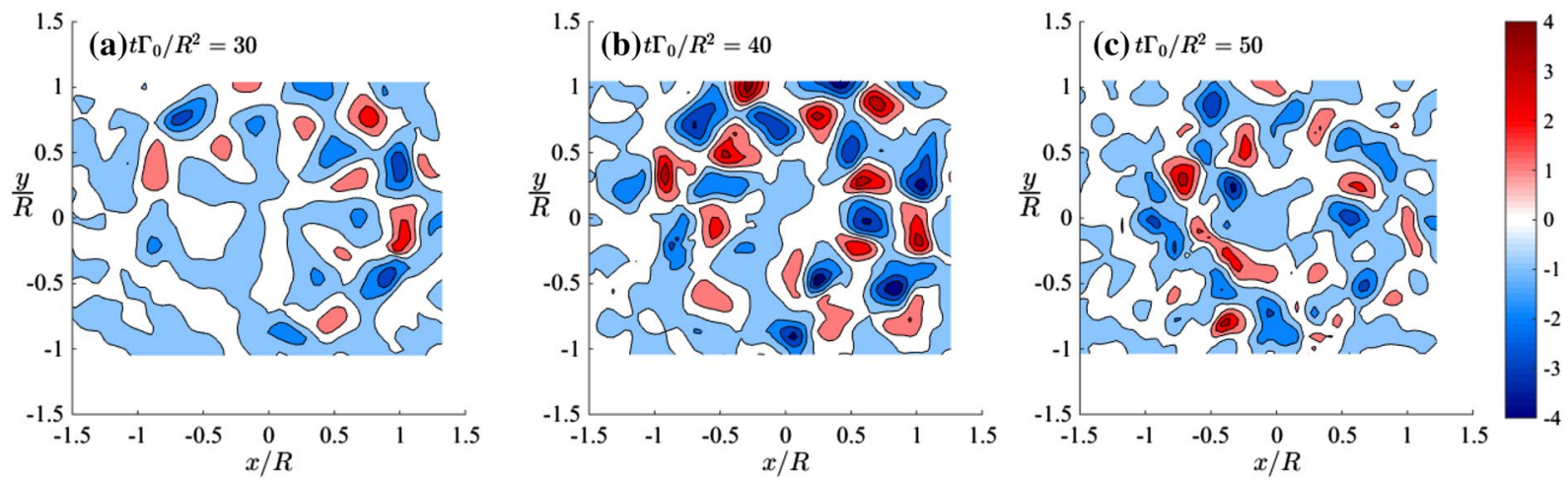

Fig. 9 Organization of axial vorticity component $\omega_{z} R^{2} / \Gamma_{0}$ in the ring median plane at $t^{*}=30,40,50$

concentric multilayer distribution of axial vorticity component was also reported by Archer et al. (2008), where three layers were observed. The third layer exterior to the ring core is associated with the secondary vortices developed outside the ring core, namely, the so-called halo-vortex. As a matter of fact, the halo-vortex is produced in the present experiment and a few cross sections can be seen in Fig. 8a and $b$. However, because of its weaker intensity and the current measurement volume, a full circular pattern is not visualized. Following the transition process, the packets of axial vorticity exhibit in-plane motion and the concentric pattern gradually becomes distorted as shown at $t^{*}=40$. Once the late stage of transition is approached, the concentric structure is completely distorted and the intensity of vorticity magnitude in the pockets is also reduced.

\subsection{Wavenumber analysis}

The tracking nature of the present experiment allows the analysis of the wavenumber evolution along the transition process. The present wavenumber analysis examines the amplitude of each wavenumber developing at the two circular positions within the median plane. One circular position has a radius $r / R=1.0$ and the other has $r / R=0.7$. Apparently, the former aims at revealing the instability taking place at the ring core, while the latter is used to reveal the secondary vortical structures. Before the extraction of the wavenumber spectrum, the axial vorticity component at the circular positions are displayed in the temporalazimuthal plane in Fig. 10. A number of 12 well-defined stripes with alternating signs show up in the transition process at both locations. The stripes at $r / R=1.0$ start to build up intensity from $t^{*}=25$, which is roughly the time when the ring undulation takes place. In the late transition stage when $t^{*}>45$, the stripes start to meander and the stripe pattern is quickly lost in the turbulent regime after $t^{*}=50$. Similar pattern can be observed at $r / R=0.7$, where secondary vortical activity happens. The delayed appearance of the stripes there agrees with the later generation of secondary vortices observed before through $3 \mathrm{D}$ isosurfaces. The organization of the stripes at $r / R=0.7$ is also subject to turbulent dissipation and the intensity is lost quickly.

The wavenumber spectrum is further calculated using the fast Fourier transformation (FFT) of the axial vorticity 

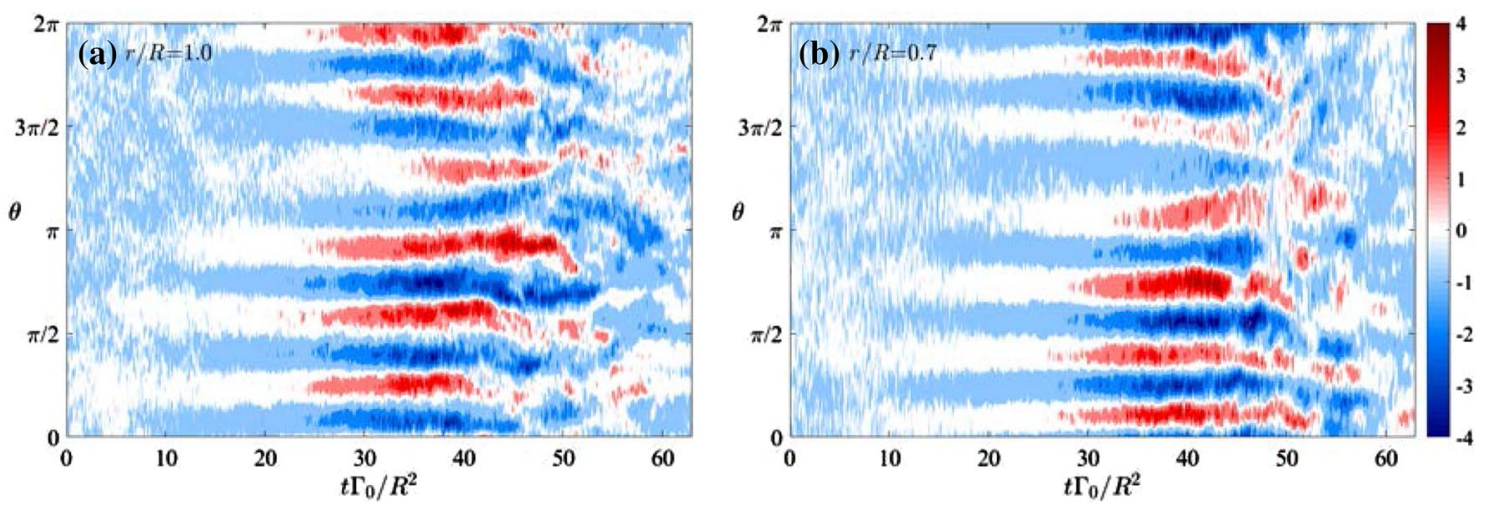

Fig. 10 Temporal evolution of axial vorticity component $\omega_{z} R^{2} / \Gamma_{0}$ in the median plane along circular lines at $r / R=1.0(\mathbf{a})$ and $r / R=0.7(\mathbf{b})$

component in Fig. 10. The spectrum results are shown in Fig. 11 in a similar fashion as Fig. 10 so as to reveal the temporal evolution. Note that due to the negligible amplitude at larger wavenumbers, only the wavenumber range of $N=1 \sim 20$ is displayed in the current analysis. In addition, note that the wavenumber amplitude is represented through the magnitude of axial vorticity. At the location of $r / R=1.0$, see Fig. 11a, strong intensity can be observed in the wavenumber range of $N=5 \sim 8$ and the peak intensity is contributed by $N=6$, which confirms the previous prediction. The transition phase is now represented by the increased wavenumber amplitude for $t^{*}=20 \sim 45$. The $N=6$ amplitude peaks at $t^{*}=40$ when the wave along the ring torus reaches its maximum. In the late transition phase, namely, $t^{*}>45$, amplitude of the wavenumbers responsible for transition decreases rapidly in intensity, and the resulted peaks are of weak magnitude and intermittency. Similar behavior can be observed for the evolution of the wavenumber spectrum associated with secondary vortical structures at $r / R=0.7$, see Fig. 11b. However, the appearance of intensified amplitude is delayed until $t^{*}=25$, which agrees with the previous observation that the instability associated with secondary vortex happens later than that at the ring core. Apart from the peak intensity at $N=6$, the amplitude of $N=7$ is of close strength especially between $t^{*}=25 \sim 35$, suggesting that two wavenumbers may dominate the secondary vortical activity.

To further examine the dominant wavenumbers in the ring core as well as in the secondary vortices, the amplitude of three prominent wavenumbers, namely, $N=5,6$, and 7 , are analyzed in terms of amplitude and phase angle. The temporal evolution of the wavenumber amplitude at the ring core is provided in Fig. 12a. Apparently, the $N=6$ wavenumber with the strongest peak is the primary wavenumber for the ring core instability, and has much larger magnitude than the other wavenumbers $N=5,7$. Moreover, it has the steepest growth rate, while the wavenumber $N=7$ has slower growth and the slowest growth rate belongs to wavenumber $N=5$. The $N=6$ wave number is apparently the dominate wave number for ring transition. It manifests as the vorticity stripes as shown earlier in Fig. 10a. The other wave numbers will interact with primary one and eventually smear the vortical structure at one corner. The growth of wavenumber amplitude is different at $r / R=0.7$,
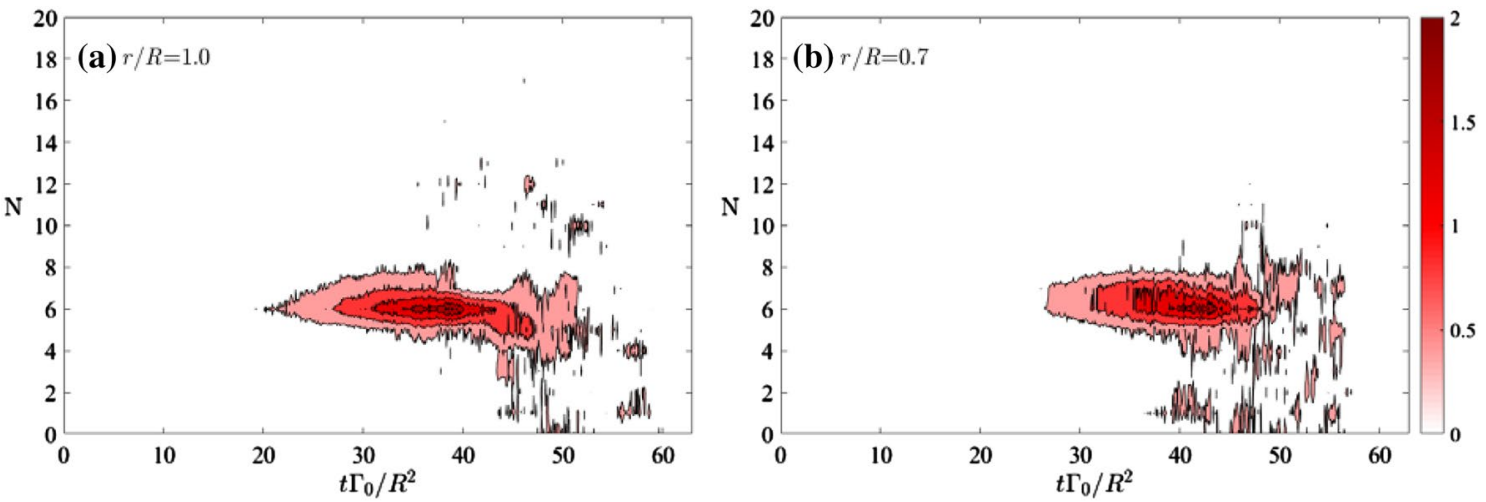

Fig. 11 Evolution of wavenumber amplitudes $\left(\omega_{z} R^{2} / \Gamma_{0}\right)$ at $r / R=1.0(\mathbf{a})$ and $r / R=0.7(\mathbf{b})$ 

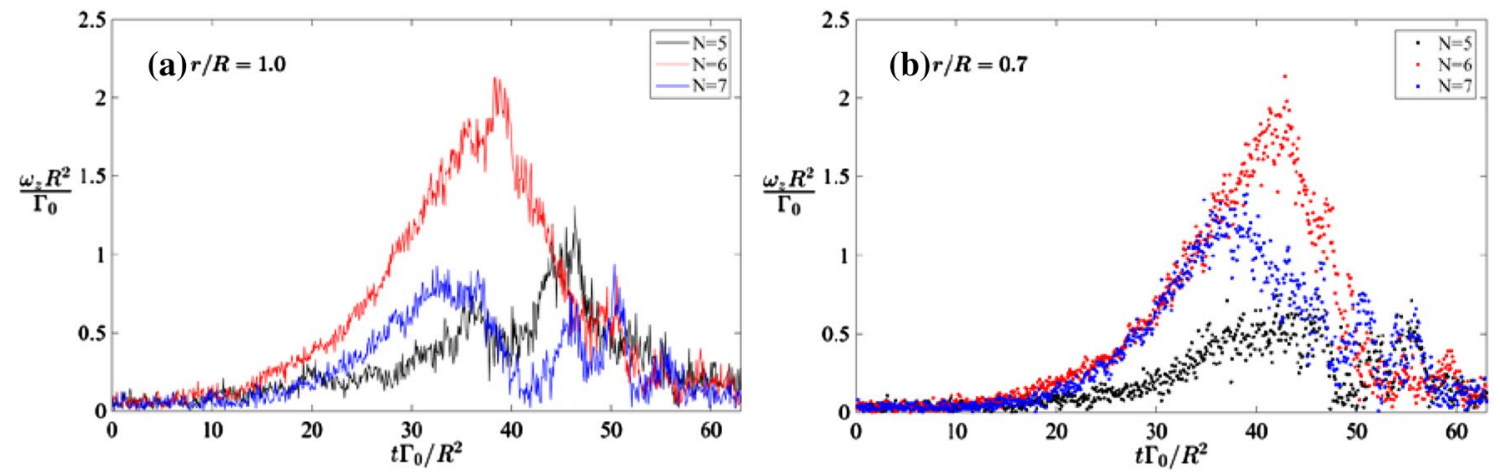

Fig. 12 Evolution of amplitudes of wavenumbers $N=5,6,7$ at $r / R=1.0$ (a) and $r / R=0.7$ (b)

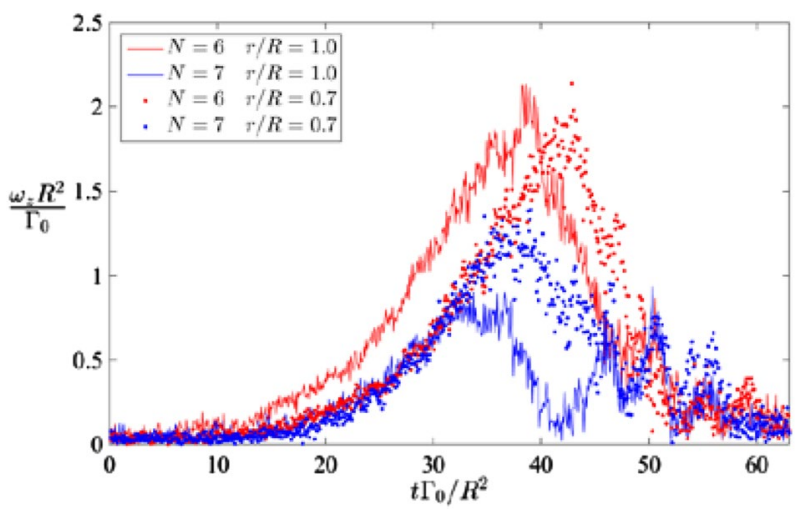

Fig. 13 Comparison of amplitude of wavenumber $N=6,7$ at $r / R=0.7$ and 1.0

see Fig. 12b. The revealed dual dominant wavenumbers of $N=6,7$ exhibit the same growth rate until $N=7$ reaches its maximum amplitude, after which the amplitude of $N=6$ keeps on growing until $t^{*}=40$. The $N=5$ wavenumber at $r / R=0.7$ also has the smallest amplitude and slowest growth among the three major wave numbers. Comparing the wavenumber amplitudes at the two locations, as presented in Fig. 13, would find that the wave number $N=7$ at ring core and the two wavenumbers $N=6,7$ for secondary vortices exhibit nearly the same growth rate although their peak amplitudes are different. The present discovery of the same growth rate of secondary wavenumber at the ring torus and primary wavenumbers associated with secondary vortices was not reported in other studies. Whether other rings of different Reynolds number and aspect ratio exhibit similar result still needs to be verified and further experiments are needed.

It is known that the instability developing at the vortex ring has the nature of a standing wave. The temporal evolution of the phase angle for the major wavenumbers of $N=5 \sim 7$ is revealed in Fig. 14. The primary wavenumber $N=6$ at the ring core has constant phase angle $\phi=0$ within the range of $t^{*}=20 \sim 45$. The wave number $N=5$, 7 both have phase angle of $\phi=\frac{1}{4} \pi$. It can be seen that the phase angle for $N=5$ is not stable and decreases slightly throughout the transition. The wavenumbers at $r / R=0.7$ have different phase difference. The dominating wavenumbers $N=6,7$ also exhibit phase difference of $\frac{1}{4} \pi$. Similarly, the phase angle of $N=5$ for the instability within the ring torus also changes. The constant phase angle of primary
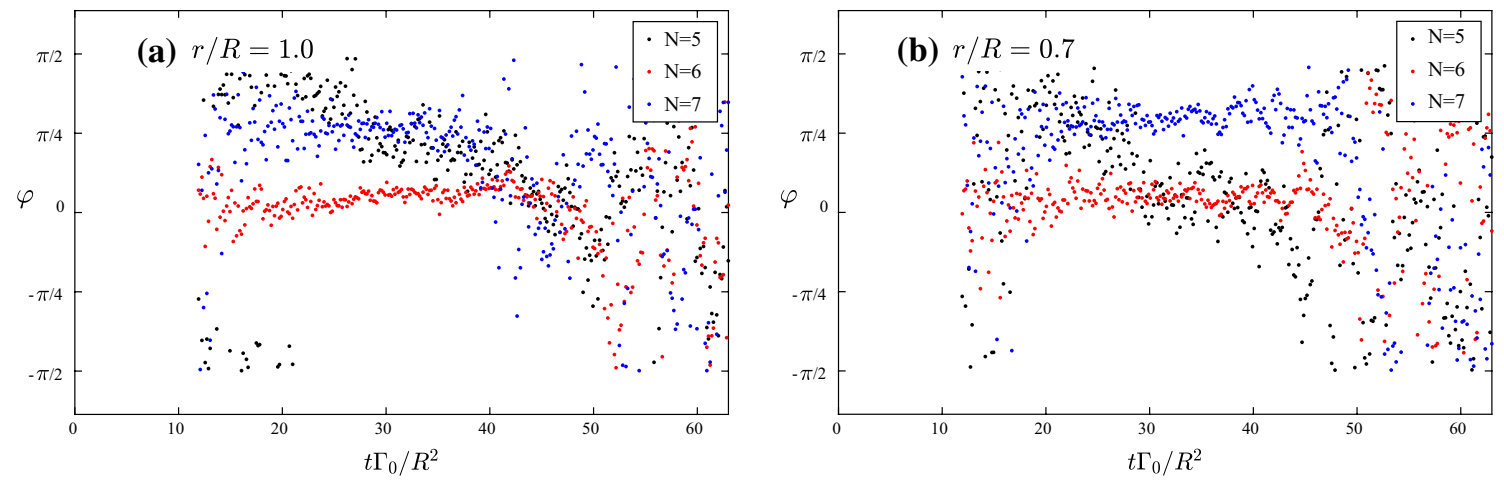

Fig. 14 Phase angle of wavenumber $N=5 \sim 7$ at $r / R=1.0$ (a) and $r / R=0.7$ (b) 
wavenumber was also verified and discussed in the experiments reported by Dazin et al. (2006).

\subsection{Transition in the Lagrangian point of view}

Further information is gained from the analysis of the particle motion in the ring. The reconstructed voxel volumes contain the particle information in the form of bright blobs representing the location of individual particles. The locations of the particle centroids are stored with subvoxel resolution through a three-axis Gaussian three-point estimator. Since a light-sheet scanning technique is used, the number of ghost particles in the reconstructed volume is largely reduced. Therefore, implementation of a simple 3D particle tracking algorithm is possible. The present algorithm leans upon the method presented by Keane et al. (1999) to reconstruct the trajectories. Note that the algorithm uses the information of the previously obtained Eulerian velocity fields to guide the search. The particle trajectories are finally smoothed with a moving average filter with a window size of six snapshots and the velocity information along the trajectories is calculated from the time-derivative. After initial stabilization of the search algorithm in the laminar phase of the ring, the tracking data were then stored from time larger than $t^{*}=8$. A representative number of 20 particle trajectories are shown in Fig. 15 through isometric view and top view. It is clear that the particles spin around the ring core which results in a periodic inwards and outwards motion in radial direction and upwards and downwards motion in the axial direction. This behavior is reflected in the plots of the history of the axial velocity $w$ and radial velocity $u_{\mathrm{r}}$ in one selected trajectory shown in Fig. 16. Both velocity components exhibit periodicity with a phase difference about $90^{\circ}$ until $t^{*}=45$ which corresponds to the end of transition. The amplitude of the axial velocity fluctuation is about 0.4 while that of the radial velocity component is lower at about 0.27 . In addition, another difference is seen in the pattern of the

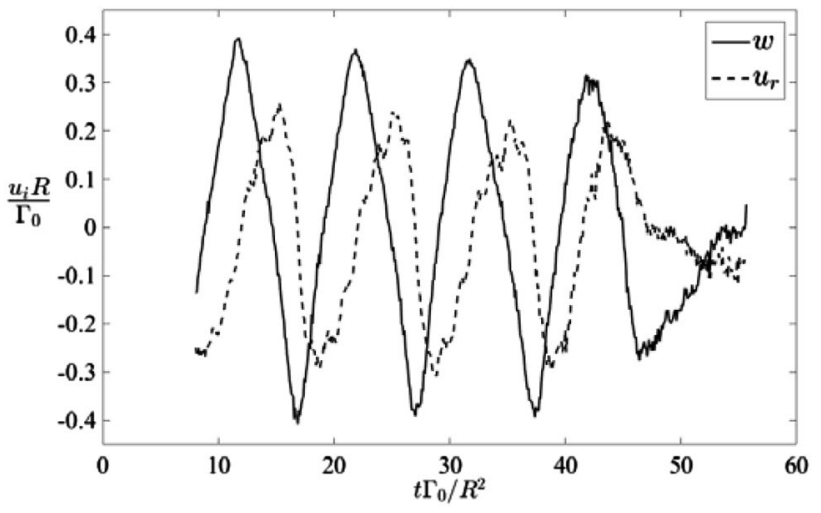

Fig. 16 History of axial velocity $w$ and radial velocity $u_{\mathrm{r}}$ along one sample particle trajectory

oscillations. While the axial component has similar slope in rise and drop between successive extrema, this is not the case for the radial component. The radial velocity slope in the rise to the maximum is weaker than its drop towards the minimum. This is referred to in the following as a skewness towards a sawtooth pattern in the radial velocity history. The clear phase relation between $w$ and $u_{\mathrm{r}}$ exists in the transition period; however, it is lost in the turbulent phase after $t^{*}=45$, which can be explained as a result of the transformation of the coherent structures into small-scale eddies. The orbital motion of the particles suggests to analyze their behavior also in the phase space spanned by the radial and axial velocity components.

The two velocity components in 200 trajectories are scatter-plotted in the $u_{\mathrm{r}}-w$ phase space in three time slots, namely, $t^{*}=10 \sim 20,20 \sim 30,30 \sim 40$, which represent the three stages before the peak amplitude of wavenumber $N=6$ is achieved. The first slot of $t^{*}=10 \sim 20$ in Fig. 17a belongs to the laminar stage, and the scattering of the individual dots is rather small. The overall structure reveals an interesting non-circular teardrop-like contour with a clear mirror-symmetric pattern to the vertical axis. A circular
Fig. 15 Twenty sample particle trajectories isometric view (a) and top view (b) (a)

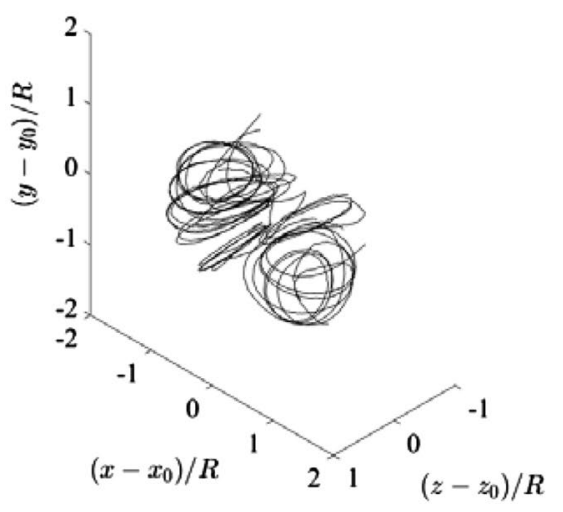

(b) 2

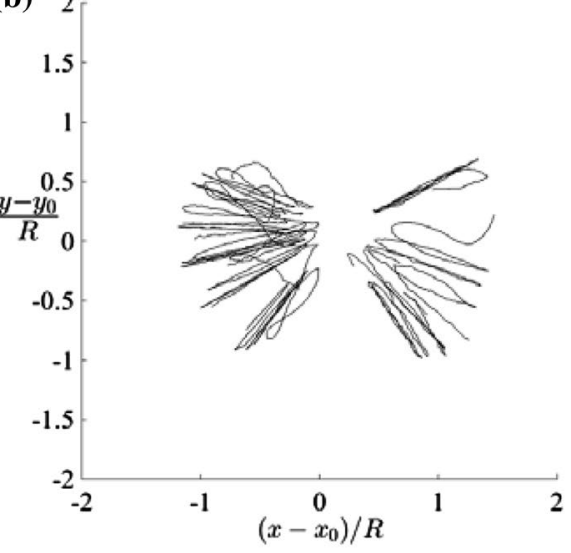



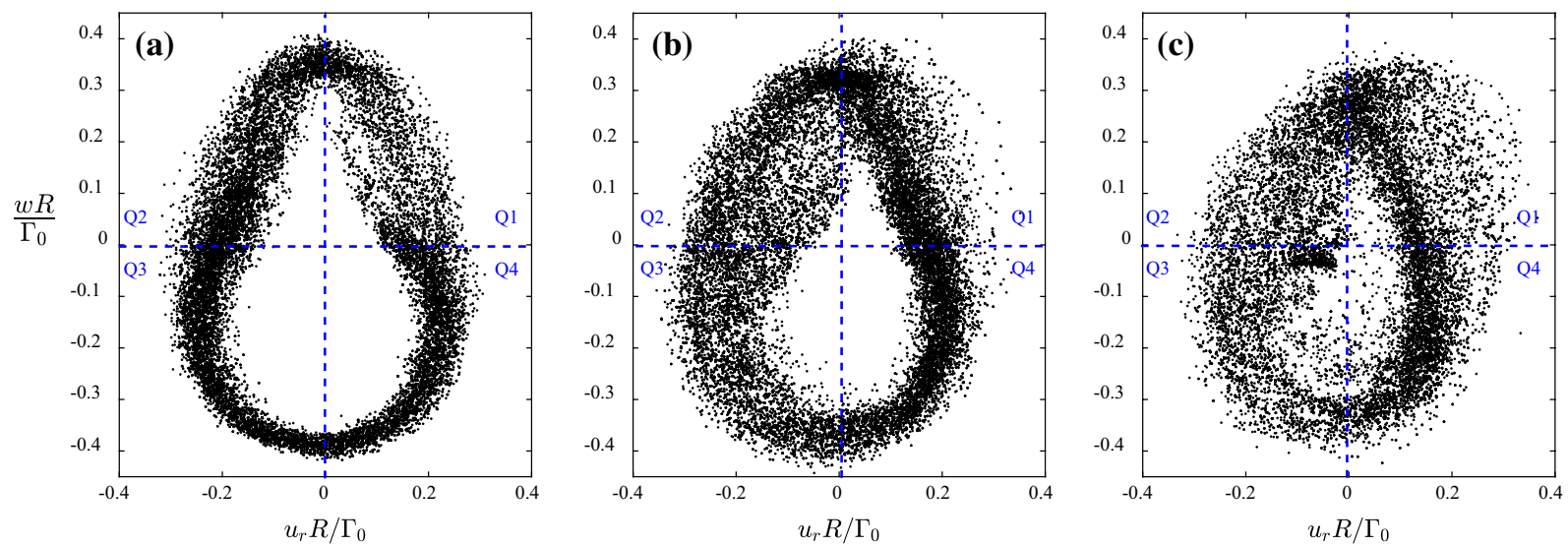

Fig. 17 Velocity polar $\left(u_{\mathrm{r}}-w\right)$ along particle trajectories at different temporal phase: $t^{*}=10 \sim 20(\mathbf{a}), 20 \sim 30$ (b), 30 40 (c)

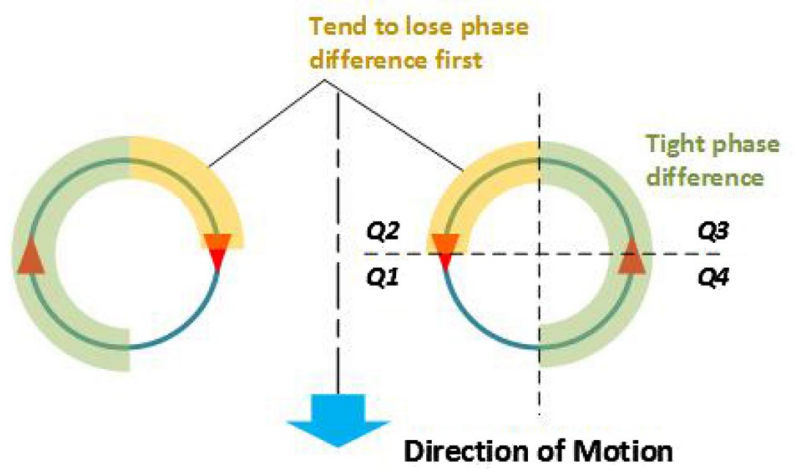

Fig. 18 Conceptual model of vortex ring for $u_{\mathrm{r}}-w$ phase relation

orbital motion of the particles would be seen as a circular scatter contour while an elliptical one would results in an elliptical contour. For better explanation of the different regions of this teardrop shape, we separate the plot in its four quadrants Q1, Q2, Q3, and Q4, as indicated in Fig. 17. Their corresponding locations in the vortex ring are given also in the conceptual model in Fig. 18. The fact that the upper part of the teardrop shape in Q1 and Q2 has a slenderer structure with a smaller horizontal width than the lower part is related to the above discussed skewness of the radial velocity profile. Because of the near $90^{\circ}$ phase difference, the rise in the profile of the radial velocity component from minimum to maximum is the phase of positive axial velocity in quadrant Q1, Q2. This is the inner region of the vortex ring, where fluid is accelerated from the aft part towards the front of the ring. In comparison, the outer region of the vortex ring in Q3 and Q4 is represented in Fig. 17a as a circular segment with small scatter. This part in Fig. 17 corresponds to the portion with $r>R$ which is characterized by a strong phase relation between both velocity components. At later times of the process in the early transition in slot $t^{*}=20 \sim 30$, the scatter along the teardrop-like contour in quadrant Q2 and Q3 increases with the tendency of the dots to move further towards the origin. This means that the radial velocity component in the aft part of the vortex ring does not reach the maximum amplitude anymore and the orbital motion there flattens in radial direction. Meanwhile, the motion pattern in Q1 and Q4 representing the front part of the vortex ring remains nearly unchanged. The results, therefore, clearly show that the aft part of the vortex ring is the location that is first subject to lose the tight phase relation. The reason for the loss of phase coherence is likely to be caused by the secondary vortical activity, which alters the coherent motion of particles induced by laminar ring. The early distortion at the inner aft portion of the ring suggests that the secondary vortices are first generated there. Following the development of transition in the slot of $t^{*}=30 \sim 40$, see Fig. $17 \mathrm{c}$, the secondary vortices are further produced, giving rise to the increase of the scatter also in quadrants Q1 and $\mathrm{Q} 4$, the front part of the ring. This is where the positive radial velocity increases in amplitude. A conceptual model in Fig. 18 is finally produced to summarize the properties associated with different portions of the vortex ring. In this model, the overlaid quadrants follow the numbering order as used in Fig. 17.

\section{Conclusions}

The present experimental study explores the transition of a vortex ring at $R e_{\Gamma}=5030$ by means of the scanning Tomo-PIV technique. The transition process is examined though various flow quantities as well as through the instability analysis. The transition in the present vortex ring is revealed to take place within $t^{*}=20 \sim 45$. The early transition for $t^{*}=20 \sim 30$ features the wavy undulation of the ring torus. The vortex ring circulation is subject to steady decrease by diffusive entrainment and the generation of 
axial and radial vorticity components is still at a slow rate. The late transition at $t^{*}=30 \sim 45$ is characterized by the generation of secondary vortices, which wrap around the undulated ring torus. In the late transition phase, the magnitudes of flow quantities including circulation and axial centroid velocity, decrease rapidly. In contrast, the axial and radial vorticity components grow fast due to vortex tilting and the generation of the secondary vortical activity.

The spectrum analysis on the axial vorticity at two circular locations corresponding to the ring torus and secondary vortices, respectively, confirms the transition lifespan. The temporal evolution of the wavenumber amplitude reveals that wavenumber $N=6$ is the dominant wavenumber for the instability developing in ring torus, while we could also observe a mode with $N=7$ related to the azimuthal arrangement of the secondary vortices. However, the amplitude of $N=6$ at the ring torus has faster growth rate. The standing wave nature for the instability is also verified in the present analysis, and the phase difference of the $N=6$ and $N=7$ modes is about $\pi / 4$ during transition.

The vortex ring is finally studied through a Lagrangian point of view, which is realized through particle tracking. The particle trajectories exhibit regular spiral motion in the early transition. The axial velocity and radial velocity along the trajectory history show a phase difference, which is revealed to be maintained in the early transition. The plot in the phase space of the radial and axial velocity shows up a well-defined teardrop-like pattern that is explained by the skewness in the evolution of the radial velocity component. This initial strong coherence is gradually lost until the end of transition. It is the inner portion of the aft of the vortex ring that is first subject to lose this coherence, which is caused by the secondary vortical events.

Acknowledgements Fundings of the position of Professor Christoph Bruecker as the BAE SYSTEMS Sir Richard Olver and Royal Academy of Engineering Chair in Aeronautical Engineering are gratefully acknowledged.

Open Access This article is distributed under the terms of the Creative Commons Attribution 4.0 International License (http:// creativecommons.org/licenses/by/4.0/), which permits unrestricted use, distribution, and reproduction in any medium, provided you give appropriate credit to the original author(s) and the source, provide a link to the Creative Commons license, and indicate if changes were made.

\section{References}

Archer PJ, Thomas TG, Coleman GN (2008) Direct numerical simulation of vortex ring evolution from the laminar to the early turbulent regime. J Fluid Mech 598:201-226

Bergdore M, Koumoutsakos P, Leonard A (2007) Direct numerical simulation of vortex rings at $R e_{\gamma}=7500$. J Fluid Mech 581:495-505

Brücker C (1997) 3D scanning PIV applied to an air flow in a motored engine using digital high-speed video. Meas Sci Technol 8:1480-1492

Dabiri J, Gharib M (2004) Fluid entrainment by isolated vortex rings. J Fluid Mech 511:311-331

Dazin A, Dupont P, Stanislas M (2006a) Experimental characterization of the instability of the vortex ring. Part II: nonlinear phase. Exp Fluids 40:401-413

Dazin A, Dupont P, Stanislas M (2006b) Experimental characterization of the instability of the vortex ring. Part I: linear phase. Exp Fluids 40:383-399

Gharib M, Rambod E, Shariff Karim (1998) A universal time scale for vortex ring formation. J Fluid Mech 360:121-140

Hegner F, Hess D, Bruecker C (2015) Volumetric 3D PIV in heart valve flow, 11th Int. Symp. Particle Image Velocimetry, Santa Barbara, USA

Keane R.D., Adrian R.J., Zhang Y. (1999) Super-resolution particle image velocimetry. Meas Sci Tech 6:754-768

Krutzsch CH (2011) On an experimentally observed phenomenon on vortex rings during their transitional movement in a real liquid. Ann Phys 532:360-379

Maxworthy T (1972) The structure and stability of vortex rings. J Fluid Mech 51:15-32

New TH, Shi S, Zhang B (2016) Some observations on vortex-ring collisions upon inclined surfaces. Exp Fluids 57:109

Pointz B, Sastuba M, Bruecker C, Kitzhofer J (2012) Volumetric velocimetry via scanning back- projection and least-squaresmatching algorithms of a vortex ring, 16th Int. Symp. on Applications of Laser Techniques to Fluid Mechanics, Lisbon, Portugal

Pointz B, Sastuba M, Bruecker C (2016) 4D visualization study of a vortex ring life cycle using modal analysis. J Vis 19(237):259

Ran H, Colonius T (2009) Numerical simulation of the sound radiated by a turbulent vortex ring. Int J Aeroacoust 8(317):336

Saffman PG (1978) The number of waves on unstable vortex rings. J Fluid Mech 84:625-639

Scarano F, Poelma C (2009) Three-dimensional vorticity patterns of cylinder wakes. Exp Fluids 47:69-83

Shariff K, Verzicco R, Orlandi P (1994) A numerical study of 3-D vortex ring instabilities: viscous corrections and early nonlinear stages. J Fluid Mech 279:351-375

Widnall S, Sullivan J (1973) On the stability of vortex rings. Proc R Soc Lond A 332:335-353 